HANS-GEORG VON ARBURG / BENEDIKT TREMP /

ELIAS ZIMMERMANN

\title{
Physiognomisches Schreiben \\ Stilistik, Rhetorik und Poetik einer gestaltdeutenden \\ Kulturtechnik
}

\section{Einleitung}

Was bedeutet es, die Welt nicht nur physiognomisch zu sehen, sondern sie auch so zu beschreiben? Oder anders gefragt: Welche Funktion hat das Schreiben und haben Erscheinungsformen der Schrift für eine jahrhundertealte Kulturtechnik, bei der sichtbare Formen und Gestaltungen als Ausdruck unsinnlicher Anlagen gedeutet werden? Dieser Frage widmet sich der vorliegende Band. Er rückt damit einen Aspekt ins Zentrum, den die wissenschaftliche Literatur zur Physiognomik bislang kaum beachtet hat. Für diese Vernachlässigung des Schreibens durch die physiognomische Theorie und ihre Analyse gibt es vor allem zwei Gründe. Entweder wird das Schreiben im Verhältnis zum physiognomischen Akt als sekundär verstanden oder aber es wird als ein unterschwelliger Anteil an diesem Akt gar nicht erst wahrgenommen. Sekundär ist das Schreiben im physiognomischen Handlungszusammenhang freilich nur dann, wenn man es als nachträgliche Verschriftlichung des zuvor physiognomisch Beobachteten und Interpretierten auffasst. Dem aber widerspricht seine subliminale Wirkung. Denn als tacit knowledge aus physiognomischen Theorien und Traktaten hat das Schreiben die physiognomische Deutung immer schon mitbestimmt.

In diesem Sinne ist das Titelbild auf dem Umschlag dieses Bandes zu verstehen: als sein programmatisches Sinnbild und als Emblem seines Erkenntnisinteresses. Schrift und physiognomische Umrisse gehen hier eine komplexe Beziehung ein. Die Unterscheidung zwischen Vordergrund und Hintergrund wird durch ihre Transparenz und gegenseitige (Ver-)Formung unklar. Auch semantisch ist das Titelbild sprechend: Kopf, Hand und Schrift sind zentrale Erkenntnisgegenstände der Physiognomik und zugleich Werkzeuge physiognomischer Aufschreibevorgänge. Die Verschriftlichung physiognomischer 'Kopfgeburten nimmt ihren Weg immer über die Hand. Physiognomisches Schreiben ist daher immer auch als ein körperlicher Prozess zu verstehen, der sich mit dem belebten und unbelebten Körper befasst und zugleich von 
ersterem zu letzterem voranschreitet. Unser Titelbild ist in diesem Sinne auf einer historischen Symbolebene zu lesen. Seine drei Komponenten sind Johann Caspar Lavaters Physiognomischen Fragmenten entnommen, als Collage und farblich modernisiert sind sie jedoch nicht mehr allein ihrer ursprünglichen Bedeutung verpflichtet. Damit reflektiert das Titelbild die Geschichte des physiognomischen Schreibens seit dem 18. Jahrhundert, in der Lavaters Ansatz zwar einen zentralen Ausgangspunkt darstellt, im Verlaufe der Moderne aber radikal verändert bzw. weiterentwickelt wurde.

Als Motto über dem vorliegenden Band reflektiert dieses Bild das formale, semantische und historische Ineinandergreifen von Physiognomik und Schreiben, das für ästhetische Formen des Schreibens ganz allgemein relevant ist. Denn literarische und künstlerische Ausdrucksformen sind dem physiognomischen Handeln generell nicht nachgeordnet, sondern konstituieren dieses wesentlich mit. In dieser Hinsicht unterhält das physiognomische Schreiben grundsätzlich zu bestimmten Formen des Wissens engere Beziehungen als zu anderen. Literatur und Kunst überhaupt bearbeiten und verarbeiten insbesondere in Konsolidierungs- und Übergangsphasen der Wissensgeschichte vorzugsweise unsicheres oder verunsichertes Wissen. In ästhetischer Hinsicht hat es das physiognomische Schreiben daher sowohl systematisch als auch historisch in signifikanter Weise mit unsicherem Wissen zu tun. Interessiert man sich für die wissensgeschichtliche Bedeutung und Funktion der Physiognomik, wie wir dies im vorliegenden Sammelband tun, dann ist die Schreibkunst für dieses unsichere Wissen allerdings nicht so sehr als Einfluss- oder Bedingungsverhältnis von Belang. Sie interessiert vielmehr als Komponente jener diskursiven Regulierungen und Modellierungen, die physiognomisches Wissen in einem gegebenen gesellschaftlichen Handlungszusammenhang formiert und artikuliert.

Das unsichere Wissen der Physiognomik hat freilich nicht nur eine ästhetische, sondern auch eine epistemische Komponente. Die prinzipielle Unsicherheit dieses Wissens entspringt der erkenntnistheoretischen Frage, ob und wie denn durch die Deutung eines Äußeren ein Inneres überhaupt erkannt werden kann. Die Anschlussfrage zu den darin involvierten Schreibverfahren lautet: Kann über dieses problematische Erkennen unproblematisch gesprochen und geschrieben werden und wenn ja wie? Blickt man in die lange (europäische) Geschichte der Physiognomik seit der griechischen Antike, dann drängt sich die Vermutung auf, dass die zunehmende Verhandlung dieses epistemologischen Problems seit der Mitte des 18. Jahrhunderts mit der philosophischen Begründung der modernen Ästhetik um 1750 einhergeht. Jedenfalls erfährt die Physiognomik mit dem allgemeinen 
Bedarf nach >Aufklärung` in der sSattelzeit einen Theoretisierungsschub, auf den Kunst und Literatur verstärkt mit der gleichzeitigen Ästhetisierung des physiognomischen Phänomens reagieren. Der vorliegende Band untersucht die Mechanismen dieser Ästhetisierungsbestrebungen physiognomischen Wissens und ihren Niederschlag in literarischen Stilfiguren, rhetorischen Mitteln und poetologischen Konzepten. Stilistik, Rhetorik und Poetik sind hierbei nicht als verschiedene Interessengebiete gegeneinander abzugrenzen, sondern stellen voneinander abhängige und zuweilen schwer trennbare Teilaspekte des physiognomischen Schreibens dar.

Für den epistemischen Komplex des physiognomischen Wissens charakteristisch ist der Verdacht auf Parawissenschaftlichkeit und Dilettantismus. Dieser Verdacht affiziert ganz unmittelbar auch das physiognomische Schreiben. Da der Physiognom spätestens seit Johann Caspar Lavaters Systematisierungsbestrebungen in einem Spannungsfeld zwischen laienhafter und wissenschaftlicher Tätigkeit auftritt, kann diese Leitdifferenz als Wegweiser für alle weiteren Überlegungen dienen. In der Geschichte der Physiognomik hatte die zunehmende Verwissenschaftlichung der physiognomischen Fragestellungen zur Folge, dass die alltagsweltliche Physiognomik immer mehr zu einer spekulativen Tätigkeit wurde. Dieses Spannungsfeld gewann an Dringlichkeit, seitdem die Physiognomik durch die Erfindung der Fotografie eine massive Szientifizierung erlebte. Die historisch gewachsene Unterscheidung zwischen laienhafter und professioneller Ausrichtung der Physiognomik lässt nun aber gerade nicht den Schluss zu, dass sich auch das physiognomische Schreiben in zwei distinkte Kategorien - das dilettantische Schreiben und das professionelle Schreiben - unterteilen ließe. Entscheidend scheint vielmehr die gegenseitige Abhängigkeit dieser beiden Aspekte zu sein. Eine weitere Grundsatzfrage des vorliegenden Bandes lautet daher, wie sich Dilettantismus und Wissenschaftlichkeit produktiv innerhalb des physiognomischen Schreibens niederschlagen.

Mit dem Begriff der 'gestaltdeutenden Kulturtechnik ‘ wird im Untertitel dieses Bandes schließlich darauf verwiesen, dass unter dem Begriff des sphysiognomischen Schreibens` neben der Schrift im engeren Sinne auch nichtschriftliche Verfahren und andere Medien in den Blick genommen werden müssen. Charakteristisch für die Kulturtechnik Physiognomik scheint es nämlich zu sein, dass physiognomische Texte sich immer direkt oder indirekt auf Bilder (Gemälde, Zeichnungen, Silhouetten, Fotografien etc.) stützen und dass diese Bilder auch formal in die Argumentationsstruktur integriert bzw. impliziert sind. Mit ihrer Hilfe werden schriftliche Beschreibungen etabliert, intensiviert, plausibilisiert oder legitimiert, so dass ihre Funktion kaum je 
eine bloß sekundäre oder komplementäre ist. Nicht selten treten Abbildungen mit der Schrift auch in Konkurrenz oder stellen diese (unwillkürlich) in Frage. Sucht man nach allgemeinen spoetischen«, 'poetologischen oder stropologischen Mustern sprachlicher wie nichtsprachlicher Äußerungsformen im Hinblick auf eine allgemeine physiognomische Stilistik, dann setzt man evidente Gemeinsamkeiten zwischen diesen Techniken voraus. Diesen Gemeinsamkeiten stehen nicht weniger gewichtige Differenzen gegenüber. In diesem Sinne lässt sich etwa nach der Uneigentlichkeit der Silhouette und ihrer ambivalenten Erkenntnisfunktion fragen, um nur ein Beispiel unter vielen zu erwähnen.

Schriftliche wie nichtschriftliche physiognomische Beschreibungstechniken üben sodann eine beachtliche Wirkung auf literarische, künstlerische und architektonische Produktionsverfahren aus, die keinen expliziten Anspruch erheben, selbst physiognomisches Wissen zu produzieren. Dennoch nehmen auch diese raußenstehenden Erzeugnisse an physiognomischen Diskursen teil und affizieren deren Theoriebildung. Während z.B. die physiognomische Analyse der gebauten Umwelt am Ende des 18. Jahrhunderts in der deutschsprachigen Architekturtheorie zum wissenschaftlichen Projekt erhoben wird, gibt es in der französischen architecture parlante schon früher Bestrebungen, in einem Gebäude den charakteristischen Ausdruck seiner Bewohner darzustellen. Physiognomische Beschreibungen und interagierende Produktionsformen gehen aber insbesondere auch in der bildenden Kunst Hand in Hand. So wurden etwa Johann Heinrich Füsslis Stiche, die in Auseinandersetzung mit Lavaters Physiognomik entstanden, ihrerseits von Physiognomen als Anschauungsmaterial herangezogen.

Die Tatsache, dass die gestaltdeutende Kulturtechnik Physiognomik eine künstlerische Gestaltproduktion hervorruft und durch diese ihrerseits wiederum angeregt wird, lässt sich als wechselseitiger Ästhetisierungs- und Szientifizierungsprozess beschreiben. Zur wissenschaftlichen Analyse dieses Prozesses bieten sich die Begriffspaare Stilistik und Gattung, Rhetorik und Tropen sowie Poetik und Schreibszenen als heuristische Leitkategorien an, mit deren Hilfe sich das weite Feld möglicher Fragen und Aspekte eingrenzen und kartieren lässt.

1. Stilistik und Gattung: Jede Stilistik ist stets an den Typus und den Erscheinungsort eines Textes geknüpft. Sie definiert sich allerdings auch über ein Spannungsverhältnis zur Gattung. Letzteres gilt besonders für den literarischen Text, besteht doch ein mögliches Merkmal von Literarizität/Poetizität in der Loslösung und Überschreitung gattungsspezifischer Regeln. 
Welchen Gattungsregeln der Stil eines physiognomischen Schreibens folgt und welche er überschreitet, ist deshalb von grundlegendem Interesse. $\mathrm{Zu}$ denken wäre hier etwa an die Wissenschaftssatire in Georg Christoph Lichtenbergs Angriffen auf Lavater oder an die essayistische Form von Walter Benjamins Passagen-Werk. Fraglich wird in diesem Zusammenhang aber auch die Gattung jener physiognomischen Texte, die um die Wissenschaftlichkeit ihrer `Disziplin` zwischen Philosophie, Esoterik und Naturwissenschaft bemüht sind - allen voran die Gattung von Lavaters Physiognomischen Fragmenten, später aber auch von Carl Huters psycho-physiognomischer Menschenkenntnis durch Körper-, Lebens-, Seelen- und Gesichts-Ausdruckskunde oder von Ludwig Klages' Prinzipien der Charakterologie. Inwiefern schreiben sich derartige Projekte in bestehende wissenschaftliche Textsorten und Textregister ein bzw. erweitern oder revidieren diese?

'Stik kann aber auch selbst als ein sphysiognomischer Begriff verstanden werden. Spätestens seit den terminologischen Bestimmungen des Renaissancetheoretikers Giorgio Vasari befindet sich der Stilbegriff in einem konstitutiven Beziehungsverhältnis zum Begriff des `Charakters ‘ und wird um 1800 von Ästhetikern wie Karl Philipp Moritz noch stärker an diesen gebunden. Ein Jahrhundert später entwickelt Heinrich Wölfflin seinen Stilbegriff in Auseinandersetzung mit architekturphysiognomischen Konzepten, und dieser physiognomische Stilbegriff wird von der Kunstwissenschaft der 1920er und 30er Jahre von Autoren wie Wilhelm Fraenger, Hans Sedlmayr und Hans Prinzhorn wieder aufgenommen und in pathologisierender Absicht reformuliert. sStik setzt sich dabei als terminus technicus einer im weitesten Sinne physiognomischen Ekphrasis durch, die das 'Ende der Physiognomik « in der Mitte des 20. Jahrhunderts überlebte.

2. Rhetorik und Tropen: Wie die Gattung für die Stilistik komplementär ist, so ist es für die Rhetorik die Lehre von den Tropen (Tropologie). Genauer sind es die Tropen von Metapher, Metonymie und Symbol, welche sich für eine Theorie des physiognomischen Schreibens als Kategorisierungsmerkmale anbieten. Als historische Grundfigur erschafft die metaphorische Physiognomik einen neuen Bedeutungszusammenhang durch den Vergleich zweier kategorial verschiedener und getrennter Entitäten und Bedeutungsinhalte. In der Auseinandersetzung mit Lavater bezweifelt Lichtenberg deshalb die Legitimität eines direkten Schlusses vom Äußeren aufs Innere und versteht die manipulierte Oberfläche als subjektiv geformte Metapher eines Wesenskerns. Die metonymische Physiognomik dagegen beruht auf bedeutungskonstituierenden Kontiguitäten. So argumentiert die physiognomische Phrenologie nach 
Franz Joseph Gall mit der physischen Berührung von Hirn und Schädel und zieht entsprechende Schlüsse von der Schädelform auf den Charakter, weil das Gehirn als Sitz des Charakters eine direkte physiologische Auswirkung auf den Kopf nahelegt. Die symbolische Physiognomik schließlich verlegt ihren Bedeutungsschwerpunkt in die sichtbare Repräsentation des Inneren. Nicht mehr der Vergleichszusammenhang steht hier im Vordergrund, sondern die physisch gegenwärtige neue Bedeutung des Abgebildeten. Damit wird die Innen-Außen-Differenz aufgeweicht. Die symbolisch-physiognomische Lesart eines Äußeren stellt zwar eine Innenreferenz her, doch dieses Innen lässt sich nicht länger als sverborgener Charakter verstehen. Im Symbol ist das immer schon da, worauf verwiesen wird.

Die Kategorisierung von physiognomischen Techniken im Sinne dieser Tropologie kann nun mit typischen rhetorischen Stilmitteln konfrontiert werden. So stellt sich beispielsweise die Frage, ob und wie die Großaufnahme im Film - insbesondere das Gesicht im filmischen Close-up - als uneigentliches 'Sprechen zu verstehen ist. Die filmische bzw. schauspielerische Mimik suggeriert den direkten Einblick in den Seelenzustand der Figur. Im Kontext der Handlung aber erweist sich allererst, ob es sich dabei um einen angeblich verlässlichen, metonymischen Ausdruck oder um undurchdringliche, potentielle Verstellung (und damit um eine metaphorische Konzeption) handelt. Eine Filmsprache kann Gesichter auch zum ästhetischen Selbstzweck machen - in theatralischen 'Gesichtslandschaften etwa - oder bestimmte Merkmale im Dienste einer überindividuellen Handlungslogik symbolisch in einer Weise aufladen, dass verlässliche Schlüsse auf ein psychisches Inneres kaum mehr möglich sind.

3. Poetik und Schreibszenen: In der Forschung bislang kaum diskutiert wurde die Frage nach den verschiedenen Szenen und Funktionen des Schreibens und der Schrift in der Physiognomik als Wissenspraxis. Dabei ist unübersehbar, dass sich solche Schreibszenen und -funktionen in wechselnden sozialen und medialen Umgebungen vervielfachen und je nachdem komplettieren oder konkurrieren. Schreiben als epistemische Schlüsseloperation in der physiognomischen Urteilsfindung, als Instrument der Evidenzsteigerung und Legitimierung in der Physiognomiktheorie und als Medium der Apologie oder Kritik in der öffentlichen Debatte um deren gesellschaftliche Relevanz und Geltung wirken hier in-, mit- und gegeneinander. Durch die Aufmerksamkeitsverlagerung von der topischen Bildfixierung auf pragmatische Schriftprozesse und -prozeduren gibt sich physiognomisches Wissen bis weit ins 20. Jahrhundert hinein als die Selbsttechnik (wieder) zu erkennen, als die 
sie sich in der Moderne von Anfang an - paradigmatisch in Lavaters Tagebüchern und in Lichtenbergs 'Sudelbüchern - formierte.

Von hier aus eröffnet die Analyse physiognomischen Schreibens auch einen kritischen Blick auf typische Autorpoetiken. Zur Debatte steht nicht nur die Frage, mit welchen rhetorischen Mitteln sich solche poetologischen Selbstbestimmungen artikulieren. Fraglich ist auch, welche Schreibprogrammatik sich jeweils hinter den physiognomischen Beschreibungen eines Autors oder einer Autorin verbirgt und wie sich umgekehrt konkrete ästhetisch-physiognomische Konzeptionen auf sein oder ihr Schreiben auswirken. Eine in dieser Hinsicht höchst reflexive Schreibprogrammatik weist beispielsweise das Werk Walter Benjamins auf. Dessen physiognomische Konzeption einer Geschichtsschreibung mit dem Ziel, Jahreszahlen ihre Physiognomie zu geben, kann nachgerade als locus classicus seiner Poetik gelten. Benjamin ist mit seiner physiognomischen Grundsatzreflexion aber bei weitem nicht allein. Auch bei Goethe, Jean Paul, Adalbert Stifter, Ernst Jünger und vielen anderen lässt sich ein physiognomisches Programm rekonstruieren und in den Diskursen ihrer Zeit verorten. Diese Programme müssen selbstverständlich nicht notwendigerweise mit den Befunden am literarischen bzw. wissenschaftlichen Text übereinstimmen. Gerade die Spannung zwischen poetologischer Selbstreflexion, literaturwissenschaftlicher Textanalyse und wissensgeschichtlicher Kontextualisierung macht eine kritische Auseinandersetzung mit den oftmals (zu) affirmativ gelesenen Poetiken überhaupt möglich.

Die im vorliegenden Band versammelten Beiträge beleuchten die rgestaltdeutende Kulturtechnik der Physiognomik aus literaturwissenschaftlicher, kunstgeschichtlicher und linguistischer Perspektive. In ihrem gemeinsamen Vorhaben, vor dem Hintergrund der skizzierten heuristischen Leitlinien den vielgestaltigen und multimedial vollzogenen Formen physiognomischen Schreibens nachzuspüren, richten sie sich dabei an vier thematischen Schwerpunkten aus, die den Band gliedern. Sie bündeln die Untersuchungs- und Erkenntnisinteressen unter den Gesichtspunkten "Schrift, Medium und Sprache«, »Fiktionen und Figuren«, »Bauten und Bilder« sowie »Bühne und Leinwand $\ll$.

Die Betrachtungen zum physiognomischen Schreiben setzen bewusst bei den Verhältnisgrößen von Schrift, Medium und Sprache (I) ein, handelt es sich dabei doch um die primären Bezugssysteme jeder Form von sSchreiben $<$ Die Schrift ist die von der Hand aufs Papier gebrachte Materialisierung der Schreibbewegung, das Medium ist der übergeordnete Träger 
physiognomischer Schreibdiskurse und die Sprache liefert das kommunikative Grundgerüst, das Schreibprozessen zugrunde liegt. Diese drei Begriffe werden im ersten Teil des vorliegenden Sammelbandes anhand von drei Fallbeispielen konkretisiert.

Mit der die Physiognomik im späten 18. Jahrhundert prägenden Schrift eröffnet der Beitrag von Hans-GEORG vON Arburg den Band. Er untersucht das physiognomische Problem der konkreten Handschrift Johann Caspar Lavaters unter medienästhetischen Gesichtspunkten. Dabei wird zum einen Lavaters Theoretisierung der manuellen Schreibtechnik beleuchtet, zum anderen ihre praktische Umsetzung im Rahmen des regen Autografenhandels, den der prominente Zürcher Gelehrte betrieb und in dessen Rahmen er seine Handschrift gezielt als Mittel zur Selbstinszenierung einsetzte. Durch diese wiederum werden »his master's characters", wie von Arburg aufzeigt, unter den Gesichtspunkten von >Autorschaft und `Authentizität‘ problematisch. Der Aufsatz von Peter Utz untersucht, wie in der Zwischenkriegszeit des 20. Jahrhunderts das deutsche Feuilleton zu einem wesentlichen Trägermedium kulturphysiognomischer Analytik wurde. Ausgehend von Texten u.a. von Alfred Polgar, Joseph Roth, Kurt Tucholsky und Karl Kraus vertieft Utz den Anspruch der ’kleinen Form‘, der eigenen Zeit buchstäblich ein "Gesicht« zu verleihen und dieses sodann physiognomisch auszuleuchten. Dabei umreißt er die besondere Verbundenheit des Mediums Feuilleton mit der Physiognomik unter den Gesichtspunkten der sprachlichen Darstellungsverfahren, der allgemeinen Medienkritik sowie der selbstbezogenen Gattungsreflexion.

In seinem linguistisch ausgerichteten Beitrag thematisiert MatThias AtTiG die in der Forschung bislang kaum beachteten Überlegungen zu einer sSprachphysiognomik in Walter Benjamins sprachtheoretischen Schriften. Benjamin attestierte der Sprache ein mimetisches Potential, d.h. die Möglichkeit, Nichtsprachliches wie etwa eine geistige Grundhaltung oder einen Denkstil an der eigenen Materialität abzuspiegeln und performativ einzulösen. Dieses vom deutschen Kulturphilosophen nur wenig systematisierte und zudem stark dem Mystischen verhaftete Konzept einer sprachlichen Physiognomie sucht Attig im Hinblick auf seine allgemeine »linguistische Operationalisierung « $\mathrm{zu}$ analysieren und weiterzudenken. $\mathrm{Zu}$ diesem Zweck rekurriert er auf zeichentheoretische Reflexionen bei Roman Jakobson und zeichnet auf deren Grundlage nach, wie diese Überlegungen nicht nur eine sprachwissenschaftliche Fundierung von Benjamins Ausführungen ermöglichen, sondern wie sie sich auch umgekehrt durch Benjamin erhellen lassen. 
Die oben erwähnte Ästhetisierung der Physiognomik in der Literatur der sattelzeit schlug sich zumal in Fiktionen und Figuren (II) nieder, welche die physiognomischen Diskurse ihrer Zeit reflektierten. Sie konstituierten ein künstlerisches Experimentierfeld, innerhalb dessen die sich mit und seit Lavater zügig szientifizierenden Wissens- und Erkenntnispostulate der physiognomischen Gestaltdeutung einer kritischen Prüfung unterzogen wurden. Dabei reflektierten die fiktionalen Inszenierungen zeitgenössischer Romane und Erzählungen die Physiognomik in erster Linie unter dem Gesichtspunkt ihrer wissenschaftlichen Validität und stellten die Möglichkeiten, die Grenzen und die Defizite ihrer epistemologischen Programmatik heraus. Eine entsprechende kritische Diskussion der Qualitäten physiognomischer Deutungstechniken im Rahmen fiktionalen Schreibens offenbart etwa Sophie La Roches Briefroman Die Geschichte des Fräuleins von Sternheim (1771), dem der Beitrag von Yulia Marfutova gewidmet ist. Der Roman liefert ein einschlägiges Beispiel dafür, wie die Physiognomik noch vor ihrer Popularisierung durch Lavater als wesentliches kulturelles Reflexionsmedium der Epoche literarisch verhandelt wurde. Dabei zeugt die Handlung von La Roches Werk in erster Linie von der eminenten Fehleranfälligkeit entsprechender gestaltdeutenden Bemühungen: Bedingt durch Perspektivgebundenheit und Interpretationsabhängigkeit ihrer jeweiligen Wahrnehmungen unterliegen die hier inszenierten Figuren im Versuch, sich gegenseitig zu `lesen<, regelmäßig groben Irrtümern.

Missverständnisse und Uneindeutigkeiten im Rahmen physiognomischer Lektüren trägt auch Jean Pauls Fragment gebliebener Roman Der Komet oder Nikolaus Marggraf (1820-1822) variantenreich zur Schau. Gemäß den Ausführungen von CORINNA SAUTER entfaltet sich hier auf der Ebene des Erzählens und des Erzählten ein weites Netz an Körper-, Schrift- und Bildzeichen, deren physiognomische Lesbarkeit zugleich supponiert und unterminiert wird. Weiter argumentiert Sauter, dass die Physiognomik im Komet einen wissenspoetischen 'Bilddiskurs konstituiert, dem nicht zuletzt eine intermediäre Reflexion von Jean Pauls eigenem Schreiben inhärent ist.

Der Aufsatz von Eike Kronshage erweitert die auf den deutschsprachigen Raum konzentrierte Perspektive des Bandes um einen exemplarischen Blick in den englischen Realismus. Anhand dreier Romane der Schriftstellerin Charlotte Brontë, Fane Eyre (1847), Villette (1853) und The Professor (1857), umreißt Kronshage die Tendenz der literarischen Physiognomik des Viktorianismus, einen exakten (natur-)wissenschaftlichen Anspruch für sich zu reklamieren, derweil die real praktizierte Physiognomik immer stärker in Misskredit geriet. Dabei zeigt er an Brontës Werk auf, wie diese fiktio- 
nalweltliche Aufwertung der Lavater'schen Kulturtechnik zur vorgeblichen Wissenschaft mit deren Instrumentalisierung zur Bestätigung und Festigung gängiger intersektioneller Diskriminierungsformen der Zeit einherging, welche wiederum die britischen Hegemonialansprüche untermauern sollten. Eine historische Grundkonstante der Praxis physiognomischen Schreibens besteht auch in ihrer Affinität zu Bauten und Bildern (III). Schon zeitgleich mit Lavater setzte eine Denkströmung ein, in deren Rahmen sich das originäre Interesse am physiologischen Äußeren belebter Menschenkörper auf dasjenige unbelebter konstruierter Objekte, z.B. auf Architekturen, Kleidungsstücke oder Alltagsgegenstände, ausgeweitet bzw. übertragen sieht. Hilfestellung zum praktischen Vollzug einer solchen Ding- und Kulturphysiognomik leisteten dabei seit je Bilder von zweierlei Disposition: zum einen Bilder in einem übertragenen Sinn, welche den ins Auge gefassten Gegenständen einen sinnhaften Ausdruck verleihen, zum anderen konkrete Erzeugnisse der bildenden Kunst, von den Stichen aus Lavaters Zeit bis hin zu den modernen Reproduktions- und Projektionsverfahren von Fotografie und Film. Beide Formen der Abbildung ermöglichten erst die physiognomische Les- und Beschreibbarkeit der unbelebten Konstruktionen.

Siegfried Kracauer und Walter Benjamin zählen zu den wichtigsten Vertretern einer Kulturphysiognomik des 20. Jahrhunderts, die zeitgeistige Lagen und Entwicklungen an Erscheinungsformen des Dinglich-Alltäglichen zu dechiffrieren und analysieren suchte. Wie in diesem Zusammenhang auch die U-Bahn in den Fokus dieser physiognomischen Kultur- und Gesellschaftskritik geriet, ist Gegenstand des Beitrags von BenEdikT TREMP. In ihm wird ausgeführt, wie dieses charakteristische infrastrukturelle Phänomen der Moderne im Erkenntnisprozess des physiognomischen Schreibens von Kracauer und Benjamin mit Bildern von sanitären Einrichtungen und mythologisierenden Höllenphantasien enggeführt wird und wie es gleichzeitig strukturell auf seine poetische Aufarbeitung zurückwirkt.

In der Zwischenkriegszeit etablierte sich in Deutschland die Fotografie als zentrales Medium der physiognomischen Großstadterschließung. Als solches trat sie mit schriftlichen Verfahren der Stadtbeobachtung in ein fruchtbares produktives Zusammenspiel, aus dem sich eine große Bandbreite der erzählerischen Möglichkeiten ergab. WOLFGANG BRÜCKLE präsentiert in seinem Beitrag eine reiche Auswahl an entsprechenden Beispielen und zeigt dabei auf, wie die Erfahrung fotografischer Arbeiten u.a. von Germaine Krull, Sasha Stone und Albert Vennemann die physiognomischen Programmatiken eines Franz Hessel, Siegfried Kracauer und Walter Benjamin anzuregen verstand. 
Mit der Untersuchung von Elias Zimmermann rückt der Diskurs einer >Architekturphysiognomik der Postmoderne in den Fokus. Zimmermann fragt danach, wie die ursprünglich von der französischen Revolutionsarchitektur des 18. Jahrhunderts angeregte Idee einer zur sphysiognomischen Schrift werdenden Architektur zwischen 1960 und 1980 und dabei besonders im Medium der Literatur und des Films adaptiert wurde. Über Analysen von Thomas Bernhards Roman Korrektur (1975) und Peter Greenaways Film The Belly of an Architect (1986) zeigt Zimmermann dabei auf, wie die einstige sozial-utopische Programmatik des architekturphysiognomischen Ansatzes hier einer individuell-historistischen Denkfigur Platz gemacht hat.

Eine Studie der unterschiedlichen Paradigmen physiognomischer Schreibszenen wäre nicht vollständig ohne die produktiven gestaltdeutenden Diskurse um bewegte Körper. Dieses Interesse an der Ausdruckskraft nonverbaler mimischer und gestischer Bewegungen, deren klassischer Ort die Lichtenberg'sche Pathognomik war, lässt allem voran Darbietungsformen der Bühne und Leinwand (IV) in den Mittelpunkt physiognomischer Betrachtungen treten. In diesem Zusammenhang untersuchen die Beiträge des vierten und letzten Themenschwerpunktes, wie seit Ende des 18. Jahrhunderts unterschiedlichste Ausprägungen des Tanzes und der Schauspielkunst - der theatralischen wie der filmischen - in den Brennpunkt physiognomischen Schreibens gerückt werden.

Den Anfang macht Claudia Schmölders mit einer weitgespannten Darstellung der 'Faszinationsgeschichter des Tanzes von Lady Hamiltons europaweit gefeierten pantomimischen >Attitüden um 1780 bis hin zum freien Ausdruckstanz der Amerikanerin Isadora Duncan um 1900. Schmölders führt aus, wie die charadenartige Rezitation antiker griechischer Vorbilder einer Hamilton oder Duncan deren zeitgenössische Betrachter zwangsläufig zu Physiognomen werden ließ, welche die Ursache hinter den dargebotenen Posen zu dechiffrieren hatten. Einschlägige Zeugnisse u.a. von Goethe, Helene von Nostitz und Hugo von Hofmannsthal stützen diese These.

Ein aufschlussreiches Kapitel einer `Faszinationsgeschichte vergleichbarer Art schrieb Franz Kafka mit seiner begeisterten Akklamation der HamletVerkörperung des Schauspielers Albert Bassermann anlässlich einer Theateraufführung Max Reinhardts von 1910. Der Beitrag von UlRICH STADLER nimmt entsprechende Mitteilungen des Schriftstellers unter die Lupe und erkennt in ihnen eine eminente Neigung Kafkas zu einer pathognomischen Wirklichkeitserfassung. Mehr noch zeigt er auf, wie Kafka die BassermannPerformance zum Anlass nimmt von Reflexionen über die unterschiedlichen 
Leistungen optischer und akustischer Wahrnehmung sowie über die Gefahren und Chancen, sich in einem fremden Gesicht zu verlieren.

Die deutsche Filmkünstlerin Lotte Reiniger, bekannt vor allem für ihren Trickfilm Die Abenteuer des Prinzen Achmed (1926), ging in den 1920er Jahren als erste das Experiment an, Schattenrisse, wie sie Lavaters Physiognomik als vorrangiges Anschauungsmaterial dienten, auf Celluloid zu bannen. Katherine Rochester gibt Einblick in entsprechende Arbeiten dieser Pionierin der Animationskunst und geht dabei der Frage nach, in welcher Weise durch das Zusammenspiel von Silhouette und der modernen Technik bewegter Bilder sich die Physiognomik zur Choreografie wandelt und wie dadurch die alte sgestaltdeutende Kulturtechnik zum Brennpunkt moderner Filmtheorien wird.

URSULA VON KeITZ schließt den Band ab mit einer filmwissenschaftlichen Betrachtung heutiger Castingprozesse, die sich entweder direkt physiognomisch oder mindestens physiognomikaffin erweisen. Sie weist auf die typologischen und charakterologischen Prämissen der bisher weitgehend unterbeleuchteten Besetzungspraxis hin, bei der die verschiedenen Akteure des Filmgeschäfts widersprüchlichen Ansprüchen Rechnung zu tragen haben. Die Verantwortlichen müssen sowohl tradierte Stereotypen als auch deren innovative Überwindung berücksichtigen und oftmals abwägen zwischen dem optimalen type für die Rolle und dem publikumswirksamen image des Schauspielers. Diese komplexen Rahmenbedingungen untersucht Keitz ausführlich an der Figur des Edward Rochester in den drei jüngsten Film-/ TV-Adaptionen von Charlotte Brontës Roman Jane Eyre.

Die Beiträge des Bandes Physiognomisches Schreiben verdanken sich der gleichnamigen Tagung des Moduls "Ästhetik. Intermedialität des Wissens in Literatur, Kunst und Wissenschaft" des ProDoc "Das unsichere Wissen der Literatur. Natur, Recht, Ästhetik«. Die Veranstaltung fand im September 2015 an der Universität Lausanne statt und wurde zusammen mit dieser Publikation durch den Schweizerischen Nationalfonds ermöglicht. Die Herausgeber danken den Tagungsteilnehmern und den Verantwortlichen des Rombach Verlages für ihre tatkräftige Unterstützung. Insbesondere möchten wir auch Mariana Prusák danken, die maßgeblich an der Konzeption und Organisation der Tagung mitgewirkt hat. 